\title{
Blunt Spleen and Liver Trauma
}

\author{
Brian G. A. Dalton ${ }^{1} \quad$ Jeff J. Dehmer ${ }^{1} \quad$ Katherine W. Gonzalez ${ }^{1}$ Sohail R. Shah ${ }^{1}$ \\ ${ }^{1}$ Department of Surgery, Children's Mercy Hospital and Clinics, Kansas \\ City, Missouri, United States \\ J Pediatr Intensive Care 2015;4:10-15. \\ Address for correspondence Sohail R. Shah, MD, MSHA, Department \\ of Surgery, Children's Mercy Hospital and Clinics, 2401 Gillham Road, \\ Kansas City, MO 64108, United States \\ (e-mail: sohailshahmd@gmail.com).
}
Abstract
Keywords
- blunt abdominal trauma
- spleen injury
- liver injury
- hepatic injury
- pediatric
- nonoperative management
- angioembolization

Blunt abdominal trauma is an important cause of pediatric morbidity and mortality. The spleen and liver are the most common abdominal organs injured. Trauma to either organ can result in life-threatening bleeding. Controversy exists regarding which patients should be imaged and the correct imaging modality depending on the level of clinical suspicion for injury. Nonoperative management of blunt abdominal trauma is the standard of care for hemodynamically stable patients. However, the optimal protocol to maximize patient safety while minimizing resource utilization is a matter of debate. Adjunctive therapies for pediatric spleen and liver trauma are also an area of ongoing research. A review of the current literature on the diagnosis, management, and follow-up of pediatric spleen and liver blunt trauma is presented.

\section{Introduction}

Trauma is the leading cause of death in children older than 1 year of age and blunt trauma is the most common mechanism. The spleen and liver account for approximately $70 \%$ of all visceral injuries caused by blunt trauma, by far the most commonly injured intra-abdominal organs. ${ }^{1}$ Injury severity of spleen and liver injury is graded by the American Association for the Surgery of Trauma injury scoring scale and is shown in - Tables $\mathbf{1}$ and $\mathbf{2}$. The primary goals after blunt splenic or hepatic injury are patient stabilization and organ function preservation. Over the last 50 years, the standard of care for spleen and liver injuries has shifted to nonoperative management. Guidelines for management of the spleen and liver trauma have been proposed. ${ }^{2}$ Modifications and challenges to this protocol have certainly been made. A review of current evidence is presented here to show current strategies for diagnosis and management of blunt hepatic and splenic trauma in the pediatric population.

\section{Diagnosis}

Abdominal computed tomography (CT) is highly sensitive, specific, and readily available for expedient diagnosis of

received

October 3, 2014 accepted after revision November 10, 2014
Issue Theme Management of Childre with Trauma in the PICU; Guest Editor: Pablo Aguayo, MD

hepatic and splenic injury. ${ }^{3,4} \mathrm{CT}$ is the best imaging modality to perform when suspicion for intra-abdominal injury exists. CT has a negative predictive value (NPV) near $100 \%$ for solid organ injury. ${ }^{5}$ However, with the significant expense and exposure to radiation, there has been an effort to minimize the use of CT. Children are at especially high risk with regards to radiation-induced malignancies. ${ }^{6}$ For this reason, algorithms have been designed to identify those patients at high and low risk for intra-abdominal injury thereby guiding abdominal CT usage while reducing unnecessary radiation exposure. In a recent series of 125 pediatric patients, after blunt trauma 97 patients underwent abdominal/pelvic CT. A total of 17 of these patients were found to have intraabdominal injury. The model proposed (a group of parameters combining examination, vitals, X-ray findings, and laboratory values, see - Table 3 ) would have predicted 16 of these injuries, only missing a grade I spleen injury. ${ }^{3}$ Another series of 99 patients incorporated abdominal ultrasound along with examination, vitals and laboratory values and arrived at an NPV of $97 \%$ and a specificity of $84 \%{ }^{7}$ This protocol was further validated in a subsequent study. ${ }^{8} \mathrm{~A}$ large prospective trial that used only history and physical examination findings identified patients at very low risk for intraabdominal injury, and therefore would not need an

Copyright $\odot 2015$ by Georg Thieme Verlag KG, Stuttgart - New York
DOI http://dx.doi.org/ 10.1055/s-0035-1554983. ISSN 2146-4618. 
Table 1 Spleen injury scale

\begin{tabular}{|c|c|c|}
\hline Grade & Injury type & Description of injury \\
\hline I & Hematoma & Subcapsular, $<10 \%$ surface area \\
\hline \multirow[t]{2}{*}{ II } & Laceration & Capsular tear, $<1 \mathrm{~cm}$ parenchymal depth \\
\hline & Hematoma & Subcapsular, $10-50 \%$ surface area, intraparenchymal, $<5 \mathrm{~cm}$ in diameter \\
\hline \multirow[t]{2}{*}{ III } & Laceration & $\begin{array}{l}\text { Capsular tear, } 1-3 \mathrm{~cm} \text { parenchymal depth that does not involve a } \\
\text { trabecular vessel }\end{array}$ \\
\hline & Hematoma & $\begin{array}{l}\text { Subcapsular, }>50 \% \text { surface area or expanding; ruptured subcapsular or } \\
\text { parenchymal hematoma, intraparenchymal hematoma } \geq 5 \mathrm{~cm} \text { or } \\
\text { expanding }\end{array}$ \\
\hline \multirow[t]{2}{*}{ IV } & Laceration & $>3 \mathrm{~cm}$ parenchymal depth or involving a trabecular vessel \\
\hline & Laceration & $\begin{array}{l}\text { Laceration involving segmental or hilar vessels producing major devascu- } \\
\text { larization }(>25 \%)\end{array}$ \\
\hline \multirow[t]{2}{*}{$\mathrm{V}$} & Laceration & Completely shattered spleen \\
\hline & Vascular & Hilar vascular injury that devascularizes spleen \\
\hline
\end{tabular}

Table 2 Liver injury scale

\begin{tabular}{|l|l|l|}
\hline Grade & Injury type & Description of injury \\
\hline \multirow{3}{*}{ I } & Hematoma & Subcapsular, $<10 \%$ surface area \\
\cline { 2 - 3 } & Laceration & Capsular tear, $<1 \mathrm{~cm}$ parenchymal depth \\
\hline II & Hematoma & Subcapsular, 10-50\% surface area, intraparenchymal, $<10 \mathrm{~cm}$ in diameter \\
\hline III & Hematoma & $\begin{array}{l}\text { Subcapsular, }>50 \% \text { surface area or expanding; ruptured subcapsular or parenchymal hematoma; } \\
\text { intraparenchymal hematoma } \geq 10 \mathrm{~cm} \text { or expanding }\end{array}$ \\
\cline { 2 - 3 } & Laceration & $>3 \mathrm{~cm}$ parenchymal depth or involving a trabecular vessel \\
\hline V & Laceration & $\begin{array}{l}\text { Parenchymal disruption involving 25-75\% of hepatic lobe or } 1-3 \text { Couinaud segments in a single } \\
\text { lobe }\end{array}$ \\
\hline VI & Laceration & $\begin{array}{l}\text { Parenchymal disruption involving }>75 \% \text { of hepatic lobe or }>3 \text { Couinaud segments within a single } \\
\text { lobe }\end{array}$ \\
\cline { 2 - 3 } & Vascular & Juxtahepatic venous injuries \\
\hline
\end{tabular}

abdominal $\mathrm{CT} .{ }^{9}$ While ultrasound and clinical examination are not quite as sensitive as CT using these algorithms, or other similar protocols, may help guide selective and efficient use of CT in suspected abdominal trauma.

Focused assessment sonography for trauma (FAST) has been used as an adjunct for diagnosis in both adult and pediatric trauma patients. A series of 107 pediatric trauma patients with suspected abdominal injury underwent FAST scan and a subsequent CT abdomen/pelvis for comparison. This study revealed 20 false negatives, but only 2 false positives. ${ }^{10} \mathrm{~A}$ similar study performed in 359 adults resulted in a sensitivity of $42 \%$, specificity of $98 \%$, and an NPV of $93 \% .^{11}$ Another series of 357 patients investigating FAST to determine clinically important free fluid in pediatric patients

Table 3 High-risk clinical variables for intra-abdominal injury

\begin{tabular}{|l|}
\hline Hypotension for age \\
\hline Abnormal abdominal examination (distention, tenderness to palpation, peritonitis, contusion) \\
\hline Aspartate aminotransferase $>200 \mathrm{U} / \mathrm{L}$ \\
\hline Amylase $>100 \mathrm{U} / \mathrm{L}$ \\
\hline Microhematuria $>5$ erythrocyte/high power field \\
\hline Hematocrit $<30 \%$ \\
\hline Abnormal chest radiograph (e.g., rib, clavicle, or scapular fracture) \\
\hline
\end{tabular}



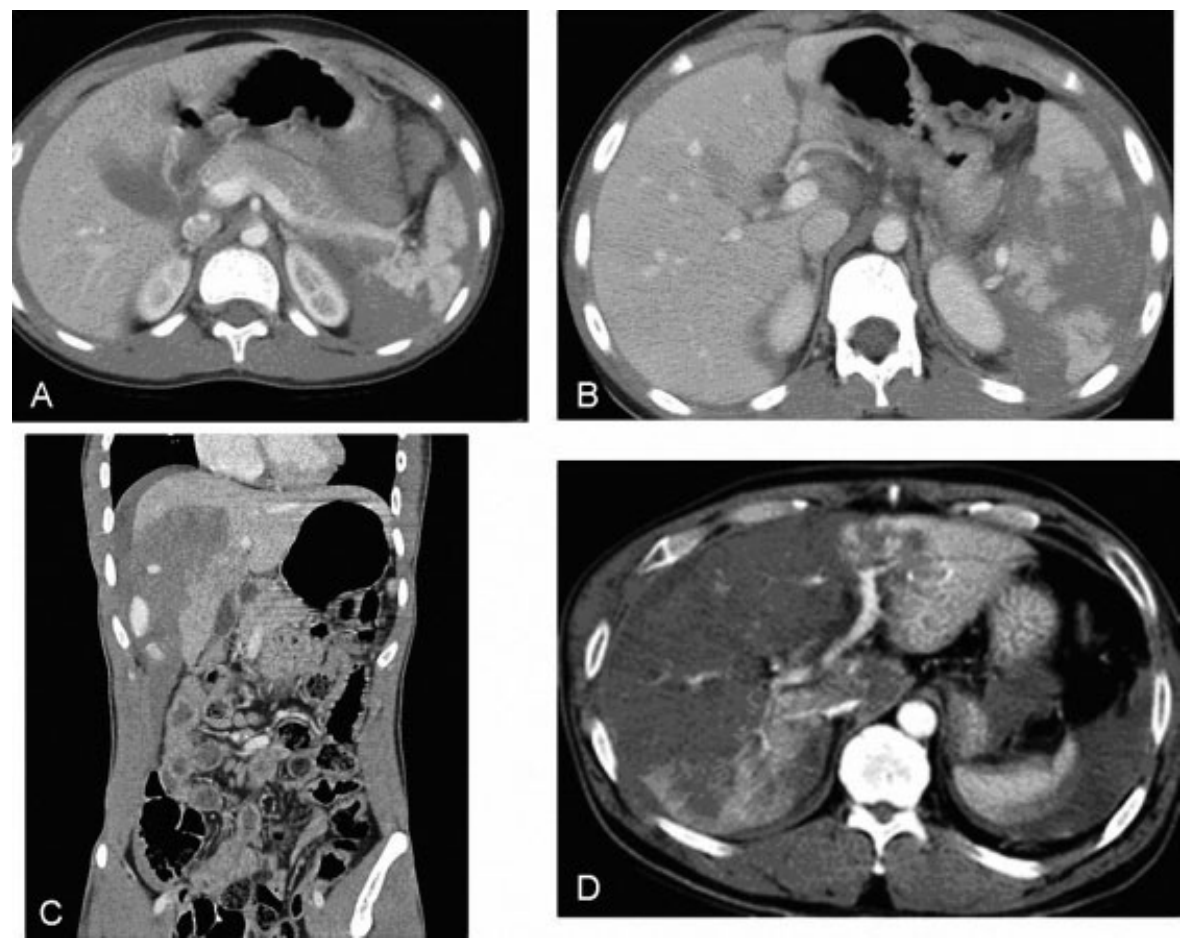

Fig. 1 Computed tomography representation of high-grade splenic and liver lacerations: (A) Grade IV splenic laceration, (B) grade V splenic laceration, (C) grade IV hepatic laceration, (D) grade V hepatic laceration.

resulted in a sensitivity of $52 \%$ and specificity of $96 \%{ }^{12}$ A more recent series of 400 pediatric trauma patients combined FAST and measurement of liver transaminases resulted in a sensitivity of $50 \%$, specificity of $98 \%$, and an NPV of $96 \%$ for the detection of intra-abdominal injuries. ${ }^{13}$ Given the low sensitivity of FAST its utility as a screening test has been called into question for hemodynamically stable pediatric patients. $^{10,11,14}$ Our current practice is to obtain a CT abdomen/pelvis on any patient with a high degree of suspicion for intra-abdominal injury given abnormal physical examination, abnormal laboratory values or other radiologic studies. We do not routinely utilize FAST as a screening tool.

\section{Management}

\section{Nonoperative versus Operative Management}

Nonoperative management of solid organ injury was first suggested in the early 1948 at the Hospital for Sick Children. ${ }^{15}$ Initial concerns regarding this approach centered on the need for increased blood transfusion, but concerns for postsplenectomy sepsis ultimately outweighed these concerns. ${ }^{16,17}$ Although it has taken almost 20 years since this initial report for surgeons to realize the benefits of solid organ preservation in the face of traumatic injury, nonoperative management of these injuries in children and adults has become universally accepted. Overall, nonoperative management has been successful in greater than $90 \%$ of patients, with splenic salvage rate of greater than $95 \%$, decreased blood transfusions and a low complication rate of less than $4 \%{ }^{18-21}$ Even high-grade injuries (grade $\geq 4$, see - Fig. $\mathbf{1}$ ) have been shown to be successfully managed by a nonoperative approach. ${ }^{21,22}$
Operative management has been restricted to hemodynamically unstable patients not responsive to resuscitation efforts.

Guidelines have been proposed for the nonoperative management of blunt splenic and hepatic trauma in children by the American Pediatric Surgery Association (APSA) trauma committee. - Table 4 shows the proposed guidelines for resource utilization in pediatric spleen and liver trauma. ${ }^{2}$ These guidelines serve as a common starting point for the management of pediatric solid organ injury, and have resulted in excellent results. ${ }^{21}$ However, recent series have challenged certain aspects of these guidelines. ${ }^{23-26}$ One prospective series of 110 patients examined an abbreviated bed rest protocol (ABRP) for spleen or liver injury. This protocol specifies one night of bed rest for grades I to II and two nights of bed rest for grade $\geq$ III. This study resulted in a mean length of stay (LOS) of 2.2 versus 3.6 days when the APSA guidelines were followed. Abdominal operations were performed in only three patients (2.3\%) and the splenic salvage rate was $98.7 \%$. After discharge from the hospital, the only activity restrictions were a 6 week omission from contact sports. No patients were readmitted for complications of solid organ injury. ${ }^{25} \mathrm{~A}$ follow-up study to this prospective trial validated the results in 199 patients and again found no readmissions related to solid organ injury and no instances of bile leak or other splenic or hepatic-related complication. ${ }^{26}$ Based on LOS data ABRP compared with APSA guidelines were theorized to potentially save over 9,000 hospital days/y and over $\$ 19$ million annually if applied nationally. ${ }^{27}$ We currently use the ABRP to guide length of bed rest orders, and recommend only restriction from contact sports for activity limitations. 
Table 4 Proposed guidelines for resource utilization in children with isolated spleen or liver injury

\begin{tabular}{|l|l|l|l|l|}
\hline Computed tomography grade & I & II & II & IV \\
\hline Intensive care unit days & 0 & 0 & 0 & 1 \\
\hline Hospital days & 2 & 3 & 4 & None \\
\hline Predischarge imaging & None & None & None & None \\
\hline Postdischarge imaging & None & None & 5 & None \\
\hline Activity restriction (wk) & 3 & 4 & 5 & 6 \\
\hline
\end{tabular}

Intensive care unit (ICU) is recommended in the setting of grade $\geq$ IV injury by the APSA guidelines. ${ }^{2}$ Other authors recommend ICU admission for patients with hemodynamic instability only, regardless of injury grade and have shown a reduction in ICU LOS without an increase in complications or operative intervention. ${ }^{24,28}$ Our practice is to admit patients that are initially hemodynamically unstable, but responsive to resuscitation to the ICU.

\section{Adjuncts to Nonoperative Management}

Complications of blunt abdominal trauma, particularly hepatic trauma, deserve special mention. In adults, complications related to hepatic trauma have been described in 11 to $24 \%$ of patients. The most common complications are biloma, hematoma, and bile leak. The vast majority of these complications can be treated with nonoperative techniques. ${ }^{29-31}$ In a series of 72 high-grade (IV-V) pediatric liver and spleen trauma patients, a mortality rate of $5.6 \%$ was found. All of these patients had liver injuries, but three of four had a severe head injury that was related to mortality. Only one patient (2.4\%) with severe spleen injury developed a complication (pleural effusion). Five patients (17.9\%) with liver injury developed complications, and all complications except one were treated with surgical adjuncts (e.g., endoscopic retrograde cholangiopancreatographic procedures, drains, transhepatic cholangiographic procedure) ${ }^{32}$ Another review of 294 patients at a single institution found 11 patients with traumatic bile leak. All patients were treated with a combination of perihepatic drain placement and endoscopic retrograde cholangiopancreatography that resulted in resolution of bile leak within 2 weeks. ${ }^{33}$ Another single-center pediatric study showed a correlation between elevated transaminase or alkaline phosphatase levels and development of complications. ${ }^{18}$ This data emphasizes the need for vigilance to assess for complications in high-grade solid organ injury, especially hepatic trauma.

\section{Angioembolization}

The role for angioembolization (AE) as a treatment for arterial hemorrhage solid organ injury unresponsive to resuscitation has been well described in adults since the $1990 \mathrm{~s} .{ }^{34}$ In adults the blush sign or contrast extravasation (CE), indicating ongoing arterial hemorrhage, has been shown to predict nonoperative failure and is an indication for angiography in many centers. ${ }^{35}$ The role of $\mathrm{AE}$ in the pediatric population is less well defined. In a series of 86 pediatric abdominal CT available for review after blunt abdominal trauma, 6 were found to have CE associated with a splenic injury. Only one of six children required surgical intervention. ${ }^{36}$ In another series of 123 pediatric patients with splenic injury, 8 were found to have CE on CT. None of these patients required intervention and patients with CE did not have a higher transfusion requirement or mortality. ${ }^{37}$ In contrast to this, a meta-analysis of pediatric spleen and liver injury revealed a lower failure rate of nonoperative management when $\mathrm{AE}$ was used a treatment option. ${ }^{38}$ In a recent single-center study of 259 pediatric patients with splenic trauma, 15 patients were treated with $\mathrm{AE}$ as the primary treatment and 8 patients were treated with $\mathrm{AE}$ after failure of observation. Only 1 patient of the 23 treated with $\mathrm{AE}$ subsequently underwent splenectomy and there were no deaths or other complications. ${ }^{39}$ However, the main indication for $\mathrm{AE}$ as treatment was $\mathrm{CE}$ and as outlined above, this indication is of questionable importance in children. Primary hepatic AE has been reporting sparsely. ${ }^{40-42}$ Although there is a small sample size, hepatic AE may act as an adjunct to nonoperative management.

More often $\mathrm{AE}$ is used to treat pseudoaneurysms that develop in a delayed fashion following blunt trauma. In a retrospective series of 362 children, the overall pseudoaneurysm rate after splenic injury was found to be 5.4 and $1.7 \%$ after hepatic injury. All were associated with grade III or IV injuries. The majority (7 of 10) of splenic artery pseudoaneurysms (SAP) closed spontaneously, but all three hepatic artery pseudoaneurysms (HAP) proceeded to embolization. ${ }^{43} \mathrm{~A}$ review of SAP found an incidence of 2 to $27 \%$ of posttraumatic patients. These patients had a high rate of intervention (75\%) but a low rate of symptoms $11 \%{ }^{44}$ Currently, there are no prospective studies in children to direct management of posttraumatic SAP or HAP.

\section{Follow-Up}

Radiologic follow-up after spleen or liver trauma in a pediatric population originally was done to assess organ healing. These radiologic tests have been able to document healing of the affected organ but have failed to consistently demonstrate an ability to detect clinically relevant complications before symptoms. Therefore, the necessity of routine follow-up, especially radiologic has been called into question. ${ }^{45-49}$ The APSA trauma guidelines do not recommend radiologic followup after a large number of patients in that series had no serious complications at follow-up. ${ }^{2}$ A prospective series in adults found six HAP in 482 patients with hepatic trauma. All 
of these patients developed gastrointestinal bleed before diagnosis. ${ }^{50}$ Without a large series with consistent followup, it is difficult to judge the exact incidence of SAP and HAP in the pediatric population. While a recent prospective trial of 199 pediatric found no complications at 10 week posthospi$\mathrm{tal}^{26}$ there have been reports of spontaneous rupture of SAP and HAP in the pediatric population. ${ }^{43,44}$ We do not currently perform any routine in-hospital or postdischarge imaging in the absence of symptoms.

\section{References}

1 Tataria M, Nance ML, Holmes JH IV, et al. Pediatric blunt abdominal injury: age is irrelevant and delayed operation is not detrimental. J Trauma 2007;63(3):608-614

2 Stylianos S; The APSA Trauma Committee. Evidence-based guidelines for resource utilization in children with isolated spleen or liver injury. J Pediatr Surg 2000;35(2):164-167, discussion 167-169

3 Streck CJ Jr, Jewett BM, Wahlquist AH, Gutierrez PS, Russell WS. Evaluation for intra-abdominal injury in children after blunt torso trauma: can we reduce unnecessary abdominal computed tomography by utilizing a clinical prediction model? J Trauma Acute Care Surg 2012;73(2):371-376, discussion 376

4 Wegner S, Colletti JE, Van Wie D. Pediatric blunt abdominal trauma. Pediatr Clin North Am 2006;53(2):243-256

5 Richards JR, Derlet RW. Computed tomography for blunt abdominal trauma in the ED: a prospective study. Am J Emerg Med 1998; 16(4):338-342

6 Brenner DJ, Hall EJ. Computed tomography-an increasing source of radiation exposure. N Engl J Med 2007;357(22):2277-2284

7 Karam O, Sanchez O, Chardot C, La Scala G. Blunt abdominal trauma in children: a score to predict the absence of organ injury. J Pediatr 2009;154(6):912-917

8 de Jong WJ, Stoepker L, Nellensteijn DR, Groen H, El Moumni M, Hulscher JB. External validation of the Blunt Abdominal Trauma in Children (BATiC) score: ruling out significant abdominal injury in children. J Trauma Acute Care Surg 2014;76(5):1282-1287

9 Holmes JF, Lillis K, Monroe D, et al; Pediatric Emergency Care Applied Research Network (PECARN). Identifying children at very low risk of clinically important blunt abdominal injuries. Ann Emerg Med 2013;62(2):107-116.e2

10 Coley BD, Mutabagani KH, Martin LC, et al. Focused abdominal sonography for trauma (FAST) in children with blunt abdominal trauma. J Trauma 2000;48(5):902-906

11 Miller MT, Pasquale MD, Bromberg WJ, Wasser TE, Cox J. Not so FAST. J Trauma 2003;54(1):52-59, discussion 59-60

12 Fox JC, Boysen M, Gharahbaghian L, et al. Test characteristics of focused assessment of sonography for trauma for clinically significant abdominal free fluid in pediatric blunt abdominal trauma. Acad Emerg Med 2011;18(5):477-482

13 Sola JE, Cheung MC, Yang R, et al. Pediatric FAST and elevated liver transaminases: An effective screening tool in blunt abdominal trauma. J Surg Res 2009;157(1):103-107

14 Scaife ER, Rollins MD, Barnhart DC, et al. The role of focused abdominal sonography for trauma (FAST) in pediatric trauma evaluation. J Pediatr Surg 2013;48(6):1377-1383

15 Upadhyaya P, Simpson JS. Splenic trauma in children. Surg Gynecol Obstet 1968;126(4):781-790

16 Ein SH, Shandling B, Simpson JS, et al. The morbidity and mortality of splenectomy in childhood. Ann Surg 1977;185(3):307-310

17 Balfanz JR, Nesbit ME Jr, Jarvis C, Krivit W. Overwhelming sepsis following splenectomy for trauma. J Pediatr 1976;88(3):458-460

18 Giss SR, Dobrilovic N, Brown RL, Garcia VF. Complications of nonoperative management of pediatric blunt hepatic injury:
Diagnosis, management, and outcomes. J Trauma 2006;61(2): 334-339

19 Davies DA, Pearl RH, Ein SH, Langer JC, Wales PW. Management of blunt splenic injury in children: evolution of the nonoperative approach. J Pediatr Surg 2009;44(5):1005-1008

20 Partrick DA, Bensard DD, Moore EE, Karrer FM. Nonoperative management of solid organ injuries in children results in decreased blood utilization. J Pediatr Surg 1999;34(11):1695-1699

21 Stylianos S. Outcomes from pediatric solid organ injury: role of standardized care guidelines. Curr Opin Pediatr 2005;17(3):402-406

22 Jim J, Leonardi MJ, Cryer HG, et al. Management of high-grade splenic injury in children. Am Surg 2008;74(10):988-992

23 Mehall JR, Ennis JS, Saltzman DA, et al. Prospective results of a standardized algorithm based on hemodynamic status for managing pediatric solid organ injury. J Am Coll Surg 2001;193(4): 347-353

24 McVay MR, Kokoska ER, Jackson RJ, Smith SD. Throwing out the "grade" book: management of isolated spleen and liver injury based on hemodynamic status. J Pediatr Surg 2008;43(6): 1072-1076

25 St Peter SD, Sharp SW, Snyder CL, et al. Prospective validation of an abbreviated bedrest protocol in the management of blunt spleen and liver injury in children. J Pediatr Surg 2011;46(1):173-177

26 St Peter SD, Aguayo P, Juang D, et al. Follow up of prospective validation of an abbreviated bedrest protocol in the management of blunt spleen and liver injury in children. J Pediatr Surg 2013; 48(12):2437-2441

27 Dodgion CM, Gosain A, Rogers A, St Peter SD, Nichol PF, Ostlie DJ. National trends in pediatric blunt spleen and liver injury management and potential benefits of an abbreviated bed rest protocol. J Pediatr Surg 2014;49(6):1004-1008, discussion 1008

28 Fremgen HE, Bratton SL, Metzger RR, Barnhart DC. Pediatric liver lacerations and intensive care: evaluation of ICU triage strategies. Pediatr Crit Care Med 2014;15(4):e183-e191

29 Carrillo EH, Spain DA, Wohltmann CD, et al. Interventional techniques are useful adjuncts in nonoperative management of hepatic injuries. J Trauma 1999;46(4):619-622, discussion 622-624

30 Christmas AB, Wilson AK, Manning B, et al. Selective management of blunt hepatic injuries including nonoperative management is a safe and effective strategy. Surgery 2005;138(4):606-610, discussion 610-611

31 Gourgiotis S, Vougas V, Germanos S, et al. Operative and nonoperative management of blunt hepatic trauma in adults: a singlecenter report. J Hepatobiliary Pancreat Surg 2007;14(4):387-391

32 Yang JC, Sharp SW, Ostlie DJ, Holcomb GW III, St Peter SD. Natural history of nonoperative management for grade 4 and 5 liver and spleen injuries in children. J Pediatr Surg 2008;43(12):2264-2267

33 Kulaylat AN, Stokes AL, Engbrecht BW, McIntyre JS, Rzucidlo SE, Cilley RE. Traumatic bile leaks from blunt liver injury in children: a multidisciplinary and minimally invasive approach to management. J Pediatr Surg 2014;49(3):424-427

34 Dent D, Alsabrook G, Erickson BA, et al. Blunt splenic injuries: high nonoperative management rate can be achieved with selective embolization. J Trauma 2004;56(5):1063-1067

35 Olthof DC, Joosse P, van der Vlies CH, de Haan RJ, Goslings JC. Prognostic factors for failure of nonoperative management in adults with blunt splenic injury: a systematic review. J Trauma Acute Care Surg 2013;74(2):546-557

36 Lutz N, Mahboubi S, Nance ML, Stafford PW. The significance of contrast blush on computed tomography in children with splenic injuries. J Pediatr Surg 2004;39(3):491-494

37 Davies DA, Ein SH, Pearl R, et al. What is the significance of contrast "blush" in pediatric blunt splenic trauma? J Pediatr Surg 2010; 45(5):916-920

38 van der Vlies CH, Saltzherr TP, Wilde JC, van Delden OM, de Haan RJ, Goslings JC. The failure rate of nonoperative management in children with splenic or liver injury with contrast blush on 
computed tomography: a systematic review. J Pediatr Surg 2010; 45(5):1044-1049

39 Gross JL, Woll NL, Hanson CA, et al. Embolization for pediatric blunt splenic injury is an alternative to splenectomy when observation fails. J Trauma Acute Care Surg 2013;75(3):421-425

40 Kiankhooy A, Sartorelli KH, Vane DW, Bhave AD. Angiographic embolization is safe and effective therapy for blunt abdominal solid organ injury in children. J Trauma 2010;68(3):526-531

41 Ong CC, Toh L, Lo RH, Yap TL, Narasimhan K. Primary hepatic artery embolization in pediatric blunt hepatic trauma. J Pediatr Surg 2012;47(12):2316-2320

42 Fallon SC, Coker MT, Hernandez JA, et al. Traumatic hepatic artery laceration managed by transarterial embolization in a pediatric patient. J Pediatr Surg 2013;48(5):E9-E12

43 Safavi A, Beaudry P, Jamieson D, Murphy JJ. Traumatic pseudoaneurysms of the liver and spleen in children: is routine screening warranted? J Pediatr Surg 2011;46(5):938-941

44 Martin K, Vanhouwelingen L, Bütter A. The significance of pseudoaneurysms in the nonoperative management of pediatric blunt splenic trauma. J Pediatr Surg 2011;46(5):933-937
45 Minarik L, Slim M, Rachlin S, Brudnicki A. Diagnostic imaging in the follow-up of nonoperative management of splenic trauma in children. Pediatr Surg Int 2002;18(5-6): 429-431

46 Benya EC, Bulas DI, Eichelberger MR, Sivit CJ. Splenic injury from blunt abdominal trauma in children: follow-up evaluation with CT. Radiology 1995;195(3):685-688

47 Huebner S, Reed MH. Analysis of the value of imaging as part of the follow-up of splenic injury in children. Pediatr Radiol 2001; 31(12):852-855

48 Lyass S, Sela T, Lebensart PD, Muggia-Sullam M. Follow-up imaging studies of blunt splenic injury: do they influence management? Isr Med Assoc J 2001;3(10):731-733

49 Rovin JD, Alford BA, McIlhenny TJ, Burns RC, Rodgers BM, McGahren ED. Follow-up abdominal computed tomography after splenic trauma in children may not be necessary. Am Surg 2001;67(2): 127-130

50 Croce MA, Fabian TC, Spiers JP, Kudsk KA. Traumatic hepatic artery pseudoaneurysm with hemobilia. Am J Surg 1994;168(3): 235-238 